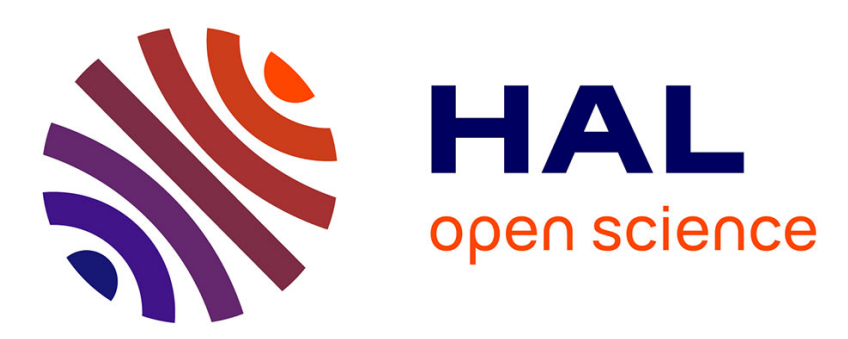

\title{
Capacity and interference modeling of CSMA/CA networks using SSI point processes
}

Anthony Busson, Guillaume Chelius

\section{To cite this version:}

Anthony Busson, Guillaume Chelius. Capacity and interference modeling of CSMA/CA networks using SSI point processes. Telecommunication Systems, 2013, 10.1007/s11235-013-9761-7 . hal00922260

\section{HAL Id: hal-00922260 \\ https://inria.hal.science/hal-00922260}

Submitted on 11 Jan 2016

HAL is a multi-disciplinary open access archive for the deposit and dissemination of scientific research documents, whether they are published or not. The documents may come from teaching and research institutions in France or abroad, or from public or private research centers.
L'archive ouverte pluridisciplinaire HAL, est destinée au dépôt et à la diffusion de documents scientifiques de niveau recherche, publiés ou non, émanant des établissements d'enseignement et de recherche français ou étrangers, des laboratoires publics ou privés. 


\title{
Capacity and Interference modeling of CSMA/CA networks using SSI point processes
}

\author{
Anthony Busson and Guillaume Chelius \\ University Paris-Sud 11 \\ Centre Scientifique d'Orsay \\ 91405 Orsay Cedex, France \\ anthony.busson@u-psud.fr \\ INRIA, ENS Lyon \\ ENS-Lyon, 69364 Lyon, France \\ guillaume.chelius@inria.fr
}

\begin{abstract}
The relative location of simultaneous transmitters, i.e. the set of nodes transmitting a frame at a given time, has a crucial impact on the performance of multi hop wireless networks. Two fundamental aspects of wireless network performances are related to these locations: capacity and interference. Indeed, as interference results from the summation of signals stemmed by concurrent transmitters, it directly depends on the transmitters' location. On the other hand, the network capacity is proportional to the number of simultaneous transmitters. In this paper, we investigate original point processes that can be used to model the location of transmitters that comply with the CSMA/CA policies, i.e. the Medium Access Control protocol used in 802.15.4 and 802.11 families of wireless technologies. We first propose the use of the Simple Sequential Inhibition point process to model CSMA/CA networks where clear channel assessment depends on the strongest emitter only. We then extend this point process to model a busy medium detection based on the strength of all concurrent signals. We finally compare the network capacity obtained through realistic simulations to a theoretical capacity estimated using the intensity of the $S S I$ point process. It turns out that the proposed model is validated by the simulations.
\end{abstract}

Keywords: multi hop wireless networks, network capacity, interference modeling, SSI point process

\section{Introduction}

The relative location of simultaneous transmitters, i.e. the set of nodes transmitting a frame at a given time, plays a crucial role in the performances of multi hop wireless networks. The CSMA/CA (Carrier Sense Multiple 
Access/Collision Avoidance) mechanism used in the IEEE 802.11 [30] and 802.15.4 [31] standards aims to control the emitters accessing the medium in order to keep a low interference level and to ensure the good reception of the frames. Thanks to CSMA/CA, two nodes transmitting a frame simultaneously cannot be very close to each other except when a collision occurs. It results in a strong spatial correlation between simultaneous transmitters' locations. Two fundamental aspects of wireless network performances are related to these locations: interference and capacity.

The performances of CSMA/CA networks in infrastructure mode has been well investigated (in [21, 34, 35, 28] for example). But, there are only a few studies dealing with the performances of the CSMA/CA mechanism in ad hoc mode, in particular about its capacity, spatial reuse and interference. The study of these quantities requires the knowledge of the transmitters' locations. In previous works, the spatial distribution of the radio nodes has generally been modeled thanks to a Poisson point process [16, 20, 3, 2, 37, 12, 14, 17, 4]. However, the Poisson point process is accurate for very sparse networks only and suffers from a lack of realism in the case of dense networks [6]. Recent works [19, 2, 26, 8] have already tackled this problem by modifying the initial Poisson process according to the mechanisms of a CSMA/CA protocol. The initial Poisson point process is thinned to select effective transmitters by applying inhibition balls around all possible transmitters. This approach leads to the well known Matérn point process [23, 24]. Other point processes as Sub-Poisson, Cluster, Neyman or Thomas point processes have also been considered [5, 19] in the literature but they do not model CSMA/CA mechanisms. However, as we will show in this paper, this approach also suffers from strong limitations. Instead, we propose to use and extend the Simple Sequential Inhibition $(S S I)$ family of point processes to alleviate these limits.

The first contribution of this paper is dedicated to the capacity estimation of CSMA/CA multi hop wireless networks. A theoretical bound on this capacity has been investigated in [17] where the authors prove that, in a network of $n$ nodes, a capacity of $\Omega\left(\frac{1}{\sqrt{n \cdot \log n}}\right)$ is feasible. In [15], authors have improved this bound and proven that an asymptotic capacity of $\Omega\left(\frac{1}{\sqrt{n}}\right)$ is feasible. In these two articles, the capacity is reached by mean of a particular transmission scheduling and routing scheme. In [13] and [25], more realistic link models have been used, both leading to a maximum asymptotic capacity of $O\left(\frac{1}{n}\right)$. In particular, authors of [25] have shown that when there is a non-zero probability of erroneous frame reception, the cumulative impact of packet losses over intermediate links results in a lower capacity. Finally, it is shown in [15], that when the path-loss function is bounded, the capacity is also $O\left(\frac{1}{n}\right)$. But these two 
last results also suppose particular transmission scheduling and routing scheme.

On the other hand, in CSMA/CA based wireless networks, the transmission scheduling is distributed and asynchronous. It is not planned in advance and depends on the link conditions, interference, etc. at the time a node wants to emit its frame. The number of simultaneous transmitters is thus highly related to the CSMA/CA mechanism which limits the spatial reuse of the channel. In other words, there is a maximum number of simultaneous transmitters in a given area and the capacity of the network is directly proportional to this number. The total number of frames sent in the whole network is thus bounded by a constant $C$ whatever the number of nodes and the routing scheme. In order to estimate the value of this constant $C$, we assess the intensity of the $S S I$ point process from a conjecture of Palásti [27]. We show through simulations that this approach gives very accurate results: intensity of the SSI fits to the number of simultaneous transmitters obtained by simulations, and the capacity deduced from this intensity corresponds to the one observed in the simulations. It empirically proves that CSMA/CA multi hop wireless networks offer a capacity of $O\left(\frac{1}{n}\right)$, and we give a very tight bound on this capacity.

The second contribution of this article concerns interference modeling. We tackle the estimation of interference distribution in dense multi hop wireless networks. As interference results from the summation of radio signals issued by concurrent transmitters, it strongly depends on the transmitters' location. We compare mean, variance and distribution of interference as function of different point processes. A similar approach has been proposed in [9], where the authors compare interference generated by different point processes (including the $S S I$ ) to simulations and known distributions, in particular heavy-tailed distributions. But some assumptions made in this thesis are inaccurate. For instance, interference distribution is estimated at an arbitrary point of the space. More realistic scenarios are considered in this paper, where interference is measured at a receiver location in the radio range of a current transmitter leading to log-normal or normal distributions rather than heavy-tailed distributions. Moreover, we proposed an extension of the S S I point process in order to take into account the first mode of the Clear Channel Assignment used in the CSMA/CA mechanism where the medium is detected busy with regard to the interference level rather than the detection of a frame.

This article is organized as follows. We first present a modeling of the radio medium and detail the CSMA/CA algorithm and its different modes in Section 2. In Section 3, we describe the point processes traditionally used in 
the literature to model the transmitters in CSMA/CA networks and highlight some of their limitations. To cope with these limitations, we present and extend the SSI point process in Section 4 and analyze it in Section 5 . We propose to use the resulting point processes to model the location of simultaneous transmitters according to the different CSMA/CA modes and we derive analytical formulae to explicit the intensity of these point processes. In Section 6 we use the intensity of the $S S I$ point process to propose a theoretical estimation of the network capacity. In Section 7. we evaluate the impact of the various point processes on the interference properties using simulations. Albeit some analytical results are available for the Matérn process [33, 7], no analytical result is yet affordable for the SS I model. Mean interference level is first evaluated and the complete interference probability density functions are then drawn and compared to classical normal and log-normal distributions. Finally, we summarize the different contributions of this article in Section 7.3 before concluding in Section 8 .

\section{Modeling CSMA/CA networks}

\subsection{Interference modeling}

When a single channel is shared by several nodes, interference is referred to as co-channel interference. For the sake of simplicity, we will restrict our work to the case of a single channel network, albeit the extension to multichannel is obvious. Let us first introduce the radio reception level $S_{j}(x)$ at a position $x$ of a signal emitted at $x_{j}$ :

$$
S_{j}(x)=l\left(\left\|x-x_{j}\right\|\right) \cdot P_{E}
$$

$P_{E}$ refers to the transmission power which we suppose constant for all nodes. $l($.) stands for the path-loss function which is related to the propagation model, and $\|$.$\| is the Euclidian norm. In most cases, l($.$) is a decreasing function$ from $R^{+}$in $R^{+}$that reflects the power decay with respect to the distance. In the simplest scenario, i.e. in line of sight (LOS), the path-loss is classically modeled using a power-law function derived from the Friis formula [29] : $l(u)=A_{0} \cdot u^{-\beta}$ where $A_{0}$ is a constant related to physical parameters - antenna gains, free-space parameter - and $\beta$ is the path-loss exponent.

To avoid near field artifact, a more realistic model is obtained by bounding this function near 0 . In the rest of the paper, numerical simulations are obtained with $l(u)=\min \left(1, \frac{A_{0}}{u^{\beta}}\right)$ with $\beta>2.0$ and $A_{0}>0$.

It is usual to consider the overall interference as a corruptive noise which affects the reception quality. In this 
case, the radio receiver performance for a transmission from node $j$ to a receiver located at $x$ is related to the Signal to Interference and Noise ratio (SINR) according to:

$$
\Gamma_{j}(x)=\frac{S_{j}(x)}{N+I_{\Phi}(x)}
$$

where $\mathrm{N}$ and I stand respectively for the receiver noise and the interference level. $I_{\Phi}(x)$ is equal to the sum of all interfering signals:

$$
I_{\Phi}(x)=\sum_{x_{i} \in \Phi ; x_{i} \neq x_{j}} S_{i}(x)
$$

where $\Phi$ is the set of concurrent transmitters. It should be noted that the radio link error probability can be directly derived from Equation 1 only if a linear receiver is used and if the interference behaves roughly like the receiver noise. This assumption may fail if the number of interferers is low or if it exists a single or few preeminent high power interferers. In this case, these strong interferers produce a correlated noise which affects in depth the performance of the receiver. If a medium access control (MAC) policy is used to prevent nearest interferers, this problem can be considered negligible.

\subsection{Medium access control}

The rules for assessing the radio medium are defined by the medium access control (MAC) protocol. In a CSMA/CA network, a candidate transmitter senses the channel before effectively transmitting. Depending on the channel state, clear or busy, the transmission is started or postponed. Clear Channel Assessment (CCA) depends on the MAC protocol and the terminal settings. For the two most widely used CSMA/CA protocols, IEEE 802.11 DCF [30] and IEEE 802.15.4 [31], CCA is performed according to one of these three methods:

1. CCA Mode 1: Energy above threshold. CCA shall report a busy medium upon detecting any energy above the Energy Detection (ED) threshold. In this case, the channel occupancy is related to the total interference level $I_{\Phi}(x)$

2. CCA Mode 2: Carrier sense only. CCA shall report a busy medium only upon the detection of a signal compliant with its own standard, i.e. same physical layer (PHY) characteristics, such as modulation or spreading. In this case, this approach is rather sensitive to the highest interfering signal rather than the overall interference level. 
Note that depending on threshold values, this signal may be above or below the ED threshold.

3. CCA Mode 3: Carrier sense with energy above threshold. CCA shall report a busy medium using a logical combination (e.g. AND or OR) of Detection of a compliant signal AND/OR Energy above the ED threshold.

An obvious consequence of the CSMA/CA modes is that two transmitters cannot be very close to each other except when a collision occurs.

\section{Classical point processes as ineffective transmitters modeling}

Generally, concurrent transmitters are modeled using the Poisson and Matèrn point processes.

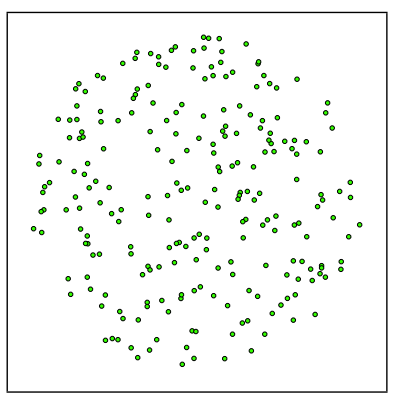

(a) Poisson ; $N=226$.

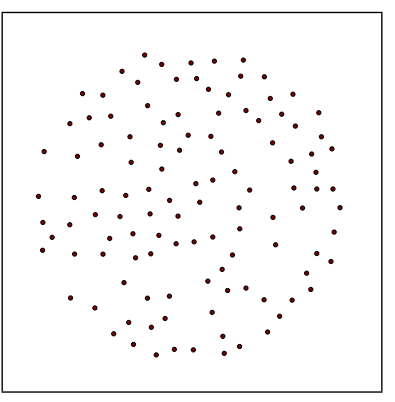

(b) Matérn ; $R_{\text {inh }}=14.9$.

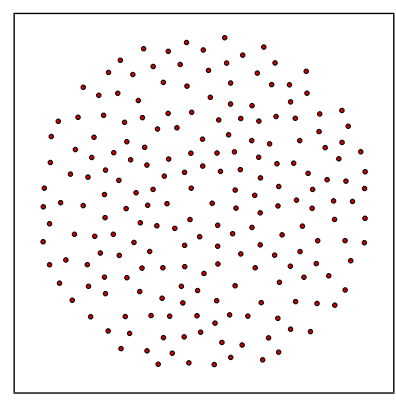

(c) $S S I ; R_{\text {inh }}=14.9$.

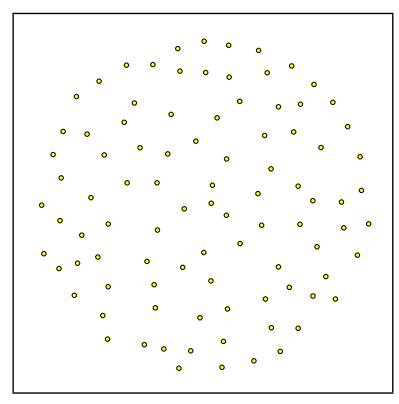

(d) $S S I_{N} ;$ see Table 5 for the parameters.

Figure 1: Samples of the different point processes. For all the point processes except the Poisson one (where $N=226), R=150.0$ and $N=1500$.

\subsection{Poisson point process}

Let us first consider the classical Poisson point process. A deep presentation of this point process can be found in [11, 33]. A sample of this point process is plotted in Figure 1(a) This process has been extensively used to model spatial distributions of active transmitters in ad hoc networks [2, 37, 12, 14, 17, 4]. One reason for this popularity is certainly the tractability of the interference distribution which is unaffordable for many other point processes. For instance, the Laplace transform of the interference distribution can be assessed and the frame error rate (FER ) can be deduced for some special cases [2]. However, the Poisson point process is only suitable to model sparse networks where transmitters can be assumed uncorrelated. In contrary, for dense networks using a CSMA/CA protocol, the MAC protocol introduces a correlation between the active transmitters location. As we will show in an upcoming section, the Poisson point process leads in fact to a very different interference distribution compared to the ones 
obtained with more realistic point processes. Consequently, the Matérn point process presented hereafter has been used to capture in some part the mechanisms of the CSMA/CA protocol.

\subsection{Matérn point process}

The Matérn point process belongs to the family of hard-core point processes, where points are forbidden to lie closer than a certain distance $h(h>0)$ to each others. The Matérn point process was introduced in [23, 24] and a convenient presentation can also be found in [33]. The Matérn point process is a dependent thinning of a Poisson point process. Thus, the set of radio nodes who stand for possible transmitters follows a Poisson point process while the distribution of instantaneous effective transmitters is modeled by a Matérn process. In the classical approach, the points of the underlying Poisson point process are deleted in such a way that the remaining points are distant of at least $h$. Thus, the minimal distance $h$ between active nodes can be seen as the maximal distance at which a node detects a compliant signal (CCA Mode 2). If the signal strength received from the closest neighbor is less than ED $\theta$, the transmission starts; otherwise it is deferred. Therefore, in numerical evaluations, the value $h$ corresponds to an inhibition radius $R_{\text {inh }}$ that can be derived from the $\operatorname{ED} \theta$ as we shall show in Section 7.1

For convenience, we rearrange the classical Matern point process definition. While in the original point process, the selection process is a function of random marks associated to each point, we propose here to use a selection process that depends on the arrival order of the points. Then the point process is built as follows:

- Let $N$ be a positive integer, let $R_{i n h}$ be an inhibition radius and let $\Phi_{M}(i)$ be the set of points selected after $i$ steps. $\Phi_{M}(0)$ is determined by the scenario as we shall see in Section 7.1

- Let $\left(X_{i}\right)_{1 \leq i \leq N}$ be a sequence of random variables uniformly distributed in $B(0, R)$, where $B(0, R)$ is the ball centered at 0 with radius $R$. The Matérn process $\Phi_{M}(N)$ is built for $i \in[1, N]$ according to:

$$
X_{i} \in \Phi_{M}(i) \text { iif }\left\|X_{i}-X_{j}\right\|>R_{\text {inh }} \forall j<i
$$

In other words, at each step, a new emitter tries to acquire the medium. If its distance to all other points is greater than $R_{\text {inh }}$, it succeeds. Otherwise the new point is kept inactive. Note however that this inactive emitter is anyway considered in the selection process of the following points. 
It is worth noting that the selection of a new point as active emitter depends on all previous points, even the inactive ones. A sample of this point process is plotted in Figure 1(b) while Figure 2(a)depicts a sample of the inhibition balls associated with a set of active transmitters selected through a Matérn point process with $N=1500$. For this value of $N$, the point process reaches saturation, i.e. no further active transmitter can be added. This figure clearly shows that even with $N$ tending to infinity, some areas remain clear of active transmitters due to the presence of inactive but inhibiting points. This observation illustrates a spatial anomaly of the Matérn process related to the fact that inactive points nevertheless impact the selection process. In the next section, a Hard Core point process is introduced to deal with this spatial anomaly. In this alternate process, the selection of a new point depends only on points previously selected as active transmitters.

\section{SSI point process as effective transmitters modeling}

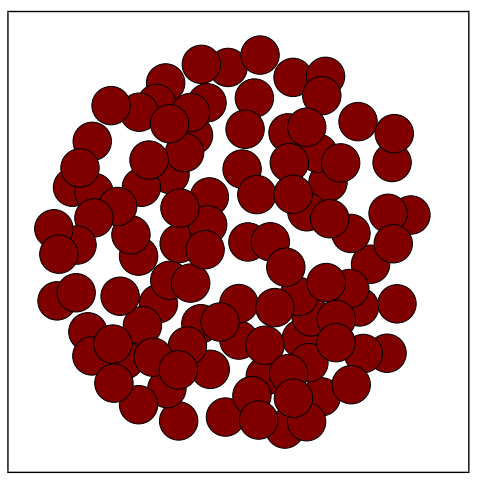

(a) Sample of a Matérn point process with its active inhibition balls.

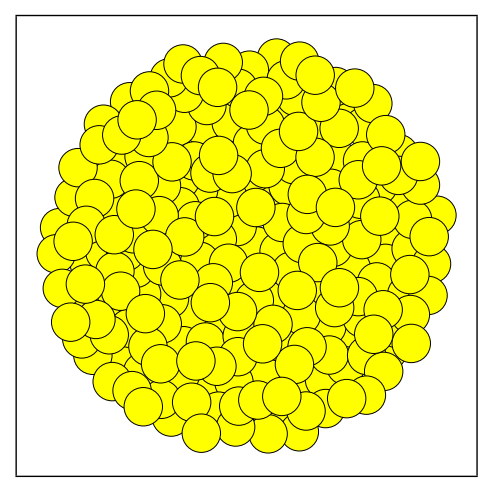

(b) Sample of a SSI point process with its inhibition balls.

Figure 2: Samples of Matérn and SSI point processes with the inhibition balls of the active transmitters. $R=150.0, N=1500$ and $h=14.9$.

The Simple Sequential Inhibition (SS I) point process was introduced by Palásti [27] and offers properties that can adequately model CSMA/CA networks. This model belongs to a family of well-known models used in the context of packing or space filling problems. They are concerned with the distribution of solids in k-dimensional spaces [18, 32]. The $S S I$ point process $\Phi_{S}(N)$ is built as follows:

- Let $N$ be a positive integer and let $\Phi_{S}(i)$ be the set of points selected in the $S S I$ after $i$ steps $\left(\Phi_{S}(0)=\Phi_{M}(0)\right)$. 
- Let $\left(X_{i}\right)_{1 \leq i \leq N}$ be a sequence of random variables uniformly distributed in $B(0, R)$. The $S S I$ process is built for $i \in[1, N]$ according to:

$$
X_{i} \in \Phi_{S}(i) \text { iif } \forall Z \in \Phi_{S}(i-1),\left\|X_{i}-Z\right\|>R_{\text {inh }}
$$

In other words, at each step, a new potential emitter attempts to acquire the medium. If its distance to all actual active emitters is greater than the inhibition radius $R_{\text {inh }}$, it becomes active. Otherwise, it is kept off, deleted from the list of transmitters and no longer considered in the selection process.

A sample of this point process is plotted in Figure 1(c) The S S I process offers a good way to model CSMA based networks implementing CCA Mode 2 as described in Section 2.2 However, as a static inhibition ball is still used, it can hardly model CCA Mode 1 . In this particular mode, the detection threshold is compared to the interference level induced by all emitters and not only the strongest one. The next model intends to address this issue.

\subsection{An extension to the Simple Sequential Inhibition $\left(S S I_{N}\right)$}

We propose an extension to the $S S I$ model denoted $S S I_{N}$. It considers the interference level generated by all neighbors in the selection process rather than the closest one only. The process is built as follows:

- Let $N$ be a positive integer. Let $\theta$ be the ED threshold of CCA Mode 1 and $\Phi_{S_{N}}(i)$ be the set of points selected in the $S S I_{N}$ after $i$ steps $\left(\Phi_{S_{N}}(0)=\Phi_{M}(0)\right)$.

- Let $\left(X_{i}\right)_{1 \leq i \leq N}$ be a sequence of random variables uniformly distributed in $B(0, R)$. The $S S I_{N}$ process is built for $i \in[1, N]$ according to:

$$
X_{i} \in \Phi_{S_{N}}(i) \text { iif } \sum_{Y_{j} \in \Phi_{S_{N}}(i-1)} l\left(\left\|X_{i}-Y_{j}\right\|\right) \cdot P_{E}<\theta
$$

In other words, at each step, a new potential emitter attempts to acquire the medium. If the interference generated by the already selected emitters is less than the ED threshold $\theta$, the transmitter becomes active. Otherwise, it is deleted and no longer considered in the selection process. Thus, the $S S I_{N}$ point process is very adequate to model CCA mode 1.

A sample of this point process is plotted in Figure 1(d). In the rest of this paper, we shall always consider the hard-core point process with $N \rightarrow+\infty$. It describes the saturated case where no further active emitter can be added. 
It is worth noting that some emitters may suffer from an interference level greater than $\theta$ at the end of the selection process. Indeed, albeit the interference level of a new emitter $X_{i}$ is bounded by $\theta$, its interference can be increased later by the selection of new transmitters $X_{j}$ with $j>i$. Note however that this phenomenon is closed to the real behavior of CSMA/CA protocols as interference is measured spatially and temporally without considering the interference increase that could result from later transmissions.

Other point processes have been recently proposed recently as Cluster, Neyman or sub-Poisson point processes [5. 19]. But they are not accurate to model CSMA/CA transmitters as they do not take into account the CSMA/CA rules in their construction.

\section{Transmitters intensity \& spatial distribution}

\subsection{Transmitters intensity}

We now investigate the transmitters intensity $\mu_{S S I_{N}}$ according to the $S S I_{N}$ point process. For the $S S I$, it has been conjectured by Palasti in [27], that

$$
\lim _{R, N \rightarrow+\infty} \frac{N_{S S I} \cdot \pi \cdot R_{i n h}^{2}}{\pi \cdot R^{2}}=4 \cdot c
$$

where $N_{S S I}$ is the number of points of the $S S I$ in $B(0, R)$ and $c$ is a constant later approximated to $c \simeq 0.56[36$, 22]. From this conjecture, we can deduce that for sufficiently large $R$ and $N$, the intensity can be estimated as:

$$
\mu_{S S I}=\frac{4 \cdot c}{\pi \cdot R_{i n h}^{2}}
$$

For our proposed point process $S S I_{N}$, we evaluate by simulation the validity of Equation 3 with a specific value of $c$ that remains to be determined. Let us write

$$
c_{N}=\lim _{N, R \rightarrow \infty} \frac{\mathbb{E}\left[N_{S S I_{N}}\right] \cdot R_{i n h}^{2}}{4 \cdot R^{2}}
$$

We use large values of $R$ and $N$ to evaluate the accuracy of this approximation. To comply with realistic assumptions, parameters are set according to the 802.15.4 and 802.11a standards.

$c_{N}$ is plotted in Figure 3(b) with $R_{i n h}$ varying from 4 to 45 meters, $\theta$ varying from $-65 d B$ to $-96 d B$ and for $\beta=3$.

The path-loss function is $l(u)=\min \left(1,\left(\frac{\lambda}{4 \pi u}\right)^{\beta}\right)$ where $\lambda$ is the wavelength. Simulations are performed in the ball $B(0, R)$ with $R=500$ meters. In these plots, $c_{N}$ appears to remain constant for all test sets and equal to $c_{N}=0.18$. As 
a consequence, these simulations show that we can experimentally estimate the intensity of the $S S I_{N}$ point processes

as

$$
\mu_{S S I_{N}}=\frac{4 \cdot c_{N}}{\pi \cdot R_{i n h}^{2}}
$$

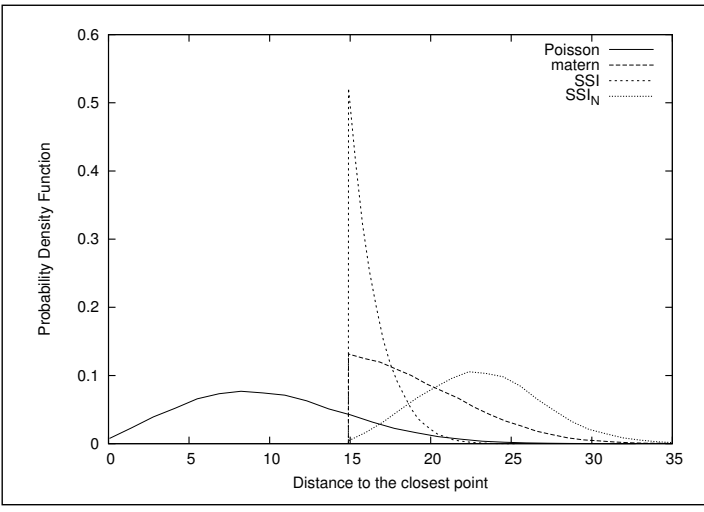

(a) Distribution of the closest interferer.

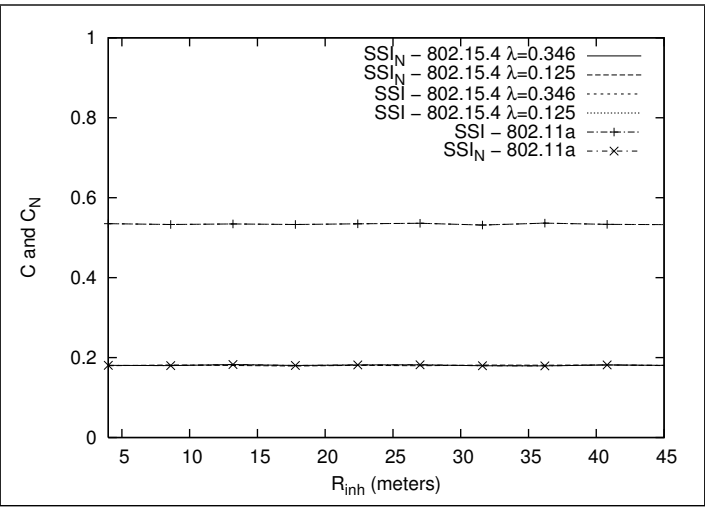

(b) $c_{N}$.

Figure 3: Distribution of the closest interferer for the different point processes and values of $c_{N}$. In the legend, $\lambda$ is the carrier wavelength.

\subsection{Transmitters spatial distributions}

The transmitter spatial distribution as modeled by the $S S I$ point process appears to be more regular than the Poisson one. Indeed, clusters of adjacent transmitters are avoided. Furthermore, the spatial distribution appears also to be more regular than the Matérn one. The problem of clear areas is avoided. This result is observed in Figure 2(b) where the inhibition balls associated with the $S S I$ point process are depicted. Contrarily to the Matérn point process, the inhibition balls cover the whole observation window. Figure 3(a) depicts the distribution of the distance between a randomly chosen point of the point process in $B\left(0, \frac{R}{2}\right)$, and its closest active neighbor. It assesses the distance distribution between an emitter and its closest interferer. These distributions have been obtained by simulations with 200000 samples. In order to avoid edge effects, the point processes are scattered in a larger window $B(0, R)$. The set of parameters are given in Table 5. Clearly, the Poisson point processes leads to a very different distribution where the points can be very close to each others compared to the other point processes. These different results induce large 
differences in the level of interference experienced by the nodes. In Section 7 , we will evaluate this impact of the different point process modelings on the resulting interference level.

\section{Capacity of CSMA/CA networks}

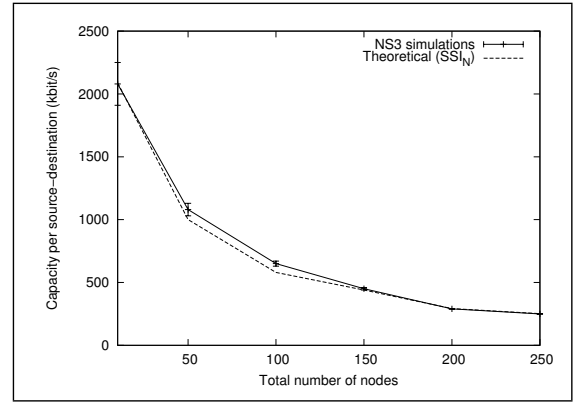

(a) One hop capacity.

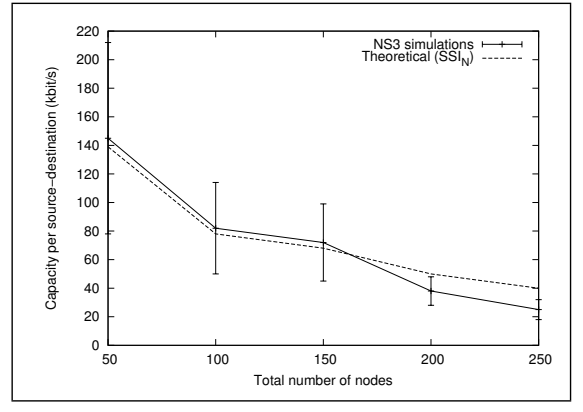

(b) Multihop capacity.

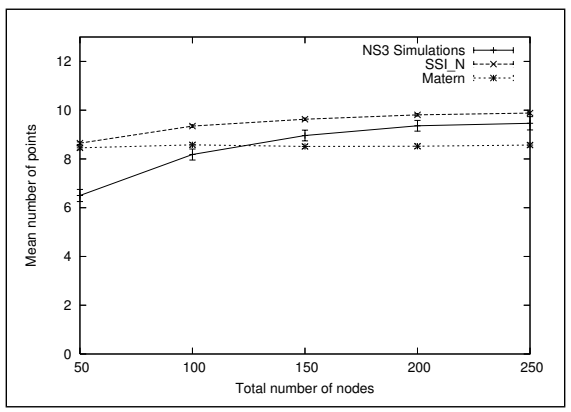

(c) Number of simultaneous transmitters.

Figure 4: Network capacity and number of simultaneous transmitters in a random Ad Hoc Network

\begin{tabular}{|l|l||l|l|}
\hline NS-3 simulation Parameters & Numerical Values & NS-3 simulation Parameters & Numerical Values \\
\hline \hline IEEE 802.11std & $802.11 \mathrm{a}$ & Path-loss function & $l(d)=\min \left(1, \frac{10^{-4.5677}}{d^{3}}\right)$ \\
CCA mode & CCA mode 1 & ED Threshold $(\theta)$ & $-99 \mathrm{dBm}$ \\
Emission power & $17.02 \mathrm{dBm}$ & Number of samples per point & 100 \\
Length of the packet & 1024 bytes & Duration of the simulation & $2 \mathrm{sec}$ \\
\hline
\end{tabular}

Table 1: Simulation parameters.

\subsection{Theoretical capacity}

We propose to take profit of the $S S I_{N}$ point process to precisely evaluate the capacity of CSMA/CA networks. Indeed, this point process seems to offer a good modeling of simultaneous transmitters. We can then estimate their 


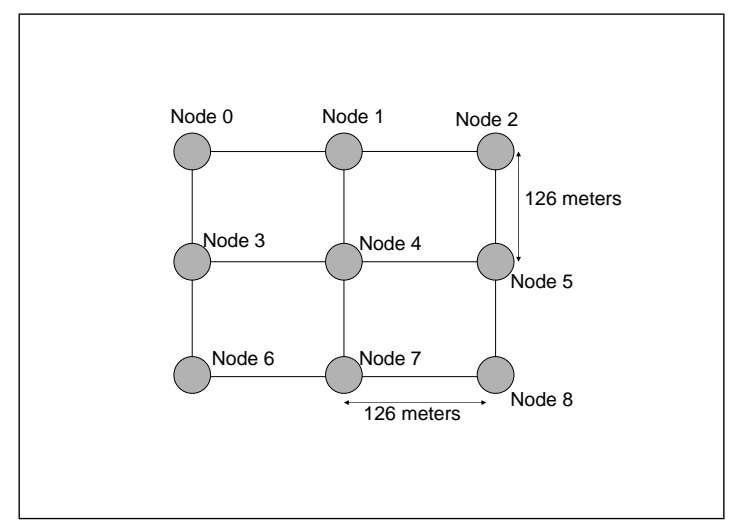

(a) The topology.

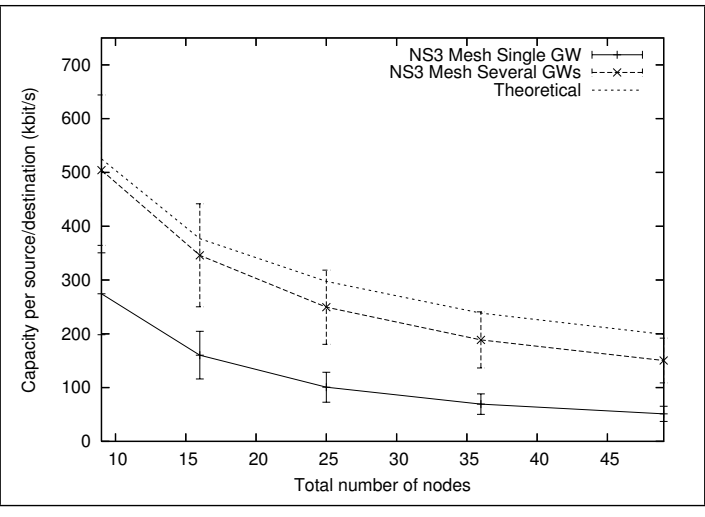

(b) Capacity.

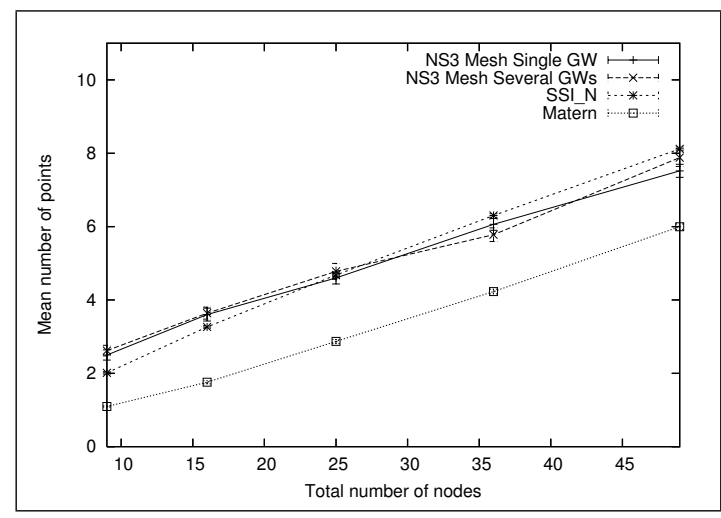

(c) Number of simultaneous transmitters.

Figure 5: Network capacity and number of simultaneous transmitters in a Mesh Network

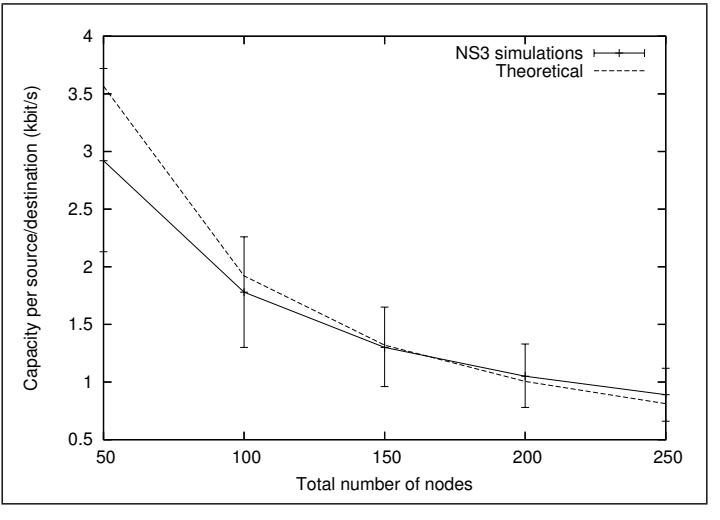

(a) Capacity.

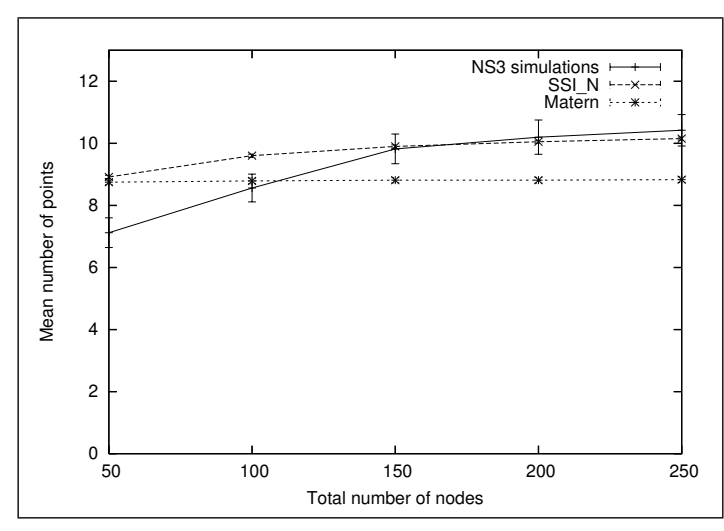

(b) Number of simultaneous transmitters.

Figure 6: Capacity and number of simultaneous transmitters in a Sensor Network 


\begin{tabular}{|l|l||l|l|}
\hline NS-3 simulation Parameters & Numerical Values & NS-3 simulation Parameters & Numerical Values \\
\hline \hline Frequency & $868 \mathrm{MHz}$ & Path-loss function & $l(d)=\min \left(1, \frac{10^{-4.567}}{d^{3}}\right)$ \\
CCA mode & CCA mode 1 & ED Threshold $(\theta)$ & $-82 \mathrm{dBm}$ \\
Emission power & $0.0 \mathrm{dBm}$ & Number of samples per point & 100 \\
Modulation & BPSK & Rate & $20 \mathrm{kbit} / \mathrm{s}$ \\
Length of the packet & 100 bytes & Duration of the simulation & $2 \mathrm{sec}$ \\
\hline
\end{tabular}

Table 2: Simulation parameters for the sensor network.

number by $\mu_{N} v(A)$ where $A$ is the observation window where the nodes are located, $v(A)$ is the corresponding area and $\mu_{S S I_{N}}$ is the intensity of the $S S I_{N}$ point process. In other words, $\mu_{S S I_{N}} v(A)$ is the mean number of points of the $S S I_{N}$ in $A$.

$$
\text { Mean number of emitters }=\mu_{S S I_{N}} \cdot v(A)
$$

The total number of frames emitted during 1 second is then $\frac{\mu_{S S I_{N} \cdot v(A)}}{\bar{T}}$, where $\bar{T}$ is the mean time to transmit a frame. If the mean size of the payload of a frame is $\bar{L}$ bits, the mean number of transmitted bits per second is then $\frac{\bar{L} \cdot \mu_{S S I_{N}} \cdot v(A)}{\bar{T}}$. We divide this quantity by the number of source-destination pairs $n$ and by the mean path length $\bar{H}$ between sources and destinations, expressed in number of hops, to obtain the final formula:

$$
\text { Capacity of the network }=\frac{\bar{L} \cdot \mu_{S S I_{N}} \cdot v(A)}{n \cdot \bar{T} \cdot \bar{H}}=\frac{C}{n}
$$

with $C=\frac{\bar{L} \cdot \mu_{S S I_{N}} \cdot v(A)}{\bar{T} \cdot \bar{H}}$.

Collisions caused by nodes transmitting at the same time is not taken into account in our model but could be introduced. For instance, in the $S S I$ selection, we could keep a point with a constant probability independently of the other points. This probability would be computed in order to represent the probability that two nodes transmit at the same time.

To validate this result, we simulate a CSMA/CA multi hop radio network using NS-3 [1]. NS-3 is a discrete-event network simulator simulating physical, link and network layers. We consider three different cases, starting with an ad hoc network where nodes are distributed in a square of size $L \times L$. In order to consider realistic scenarios [10], we 
also simulate a mesh and a sensor network. For the simulations with NS-3, each point is associated with a confidence interval of $95 \%$ in the Figures.

\subsection{Capacity of ad hoc networks}

We suppose that nodes are uniformly distributed in the observation window, a square of size 1000x1000 meters. Each node is equipped with a $802.11 \mathrm{a}$ card and configured with a constant rate of $6 \mathrm{Mbit} / \mathrm{s}$. The other simulation parameters are presented in Table 1 and in the two next paragraphs.

\subsubsection{One hop capacity}

Given the simulation parameters, the radio range of a node is approximately 150 meters and the inhibition radius $R_{\text {inh }}$ is about 205 meters. Each node is a source and emits a CBR traffic to a destination chosen randomly in its neighborhood. There is only one hop. The destination answers with the same CBR traffic, i.e. the traffic is bidirectional. In order to saturate the network, CBR flow rates are chosen close to the capacity of the links. The obtained capacity is the mean number of bits received by one node during one second.

The simulation results are plotted in Figure 4(a) and compared to the results obtained with formula (7). As we consider the CCA Mode 1, we use the intensity of the $S S I_{N}$ to evaluate the number of simultaneous transmitters. The $S S I_{N}$ intensity is evaluated numerically. As we can see, the two capacities, the one obtained with NS-3 and the theoretical one, fit perfectly well whatever the number of nodes.

\subsubsection{Multihop capacity}

In Figure 4(b), we compare the theoretical capacity obtained with formula (7) to simulation results. The scenario is the same as the previous one, except that sources and destinations are chosen randomly among all the nodes. A node is either a source or a destination. Therefore the paths between the sources and the destinations contain several hops. We use static routing to avoid any routing control traffic and routing convergence problems which occur when the network gets congested. In the theoretical estimation, the value of $\bar{H}$ is taken from simulations. The theoretical capacity fits very well the one obtained by simulations, except when the number of nodes reaches 200 . For this network cardinality, we observe a light difference caused by collisions and losses due to congested buffers. It corresponds to extreme conditions where the network is overloaded: 200 nodes scattered in 1000x1000 meters, each one generating

a CBR traffic and forwarding traffic for the other nodes. We also plotted, in Figure 4(c), the simultaneous number of 
transmitters obtained with NS-3: at a given time, we count the number of nodes that are transmitting a frame. This number is compared to the theoretical number of transmitters, i.e. the $S S I_{N}$ and the Matèrn intensities multiplied by the area of the observation window $(L \times L)$. The figure shows that the $S S I_{N}$ is the good point process to model the simultaneous transmitters as it fits very well to simulations. When the number of nodes is small, the difference between the two curves is due to the fact that, in our simulations, we considered only connected topologies. It favors topologies where nodes are gathered in a sub area of the observation window. When the number of nodes increase, all random topologies get connected and this problem disappears.

\subsection{Capacity of mesh networks}

Mesh networks are particular ad hoc networks where a set of wireless routers are deployed in order to ensure coverage and connectivity to a set of users. The mesh routers use the ad hoc mode to carry data from the users to one or several gateways connected to a wired network or to the Internet. In our scenario, mesh routers form a grid as shown in Figure 5(a) Mesh routers are in the radio range of their 2, 3 or 4 direct neighbors (2 for mesh routers on the corners, 3 for mesh routers on the edge and 4 for the others). Grids with $n \times n$ routers, $n$ varying from 3 to 7 , are considered. We do not simulate users because we assume that they are connected in infrastructure mode to the mesh routers. Mesh routers have two wireless cards: one in infrastructure mode associated to the users, and one in ad hoc mode used to carry the traffic to the gateways. The channel used by the users and the one used by the mesh networks are supposed orthogonal and do not interfere with each others. Therefore, links between users and mesh routers does not affect the capacity of the mesh network. We considered two cases: a case with a single gateway, the node 0 in the figure, and a case with several gateways, where all the nodes of the first line are gateways (nodes 0,1 and 2 in the figure). A CBR traffic, similar to the one of the ad hoc scenario, is sent from the mesh routers to the gateway(s). Other parameters are the same as in the ad hoc scenario, and are given in Table 1

In Figures 5(b) and 5(c), we show the mean number of simultaneous transmitters and the network capacity when the number of mesh routers varies. The size of $L$ increases in such a way that the distance between mesh routers remains constant (a little bit less than the radio range: 126 meters). Clearly, the $S S I_{N}$ intensity gives a very good estimate of the number of simultaneous transmitters leading to an accurate bound for the capacity. Nevertheless, it appears that this estimation is only valid when there are several gateways. Indeed, with a single gateway the bottleneck 
is the last link to this gateway and not the spatial reuse of the medium.

\subsection{Sensor networks}

We also consider a sensor network topology. We have implemented in NS-3, the physical layer of the 802.15.4 standard. All the different building blocks (BPSK modulation, data Rate, propagation model for this frequency, CSMA/CA, etc.) were already available in the simulator. The different parameters for these simulations are given in Table 2. Nodes are uniformly distributed in a square of size $75 \times 75$ meters $^{2}$. We perform simulations where each node communicates with one of its direct neighbors. Results are shown in Figures 6(a) and 6(b) Number of simultaneous transmitters and capacity fit very well to simulations. For the capacity, it offers a good estimate of the amount of data which can be exchanged between sensors. We also tested a scenario where all the traffic from the sensors have the same destination, a single sink gathering data from the sensors. The results are not shown in the figures because our approach is not pertinent in this case to estimate the capacity. As for the Mesh network with a single gateway, the capacity is limited by the wireless link to the sink and not by the spatial reuse of the medium.

\section{Interference}

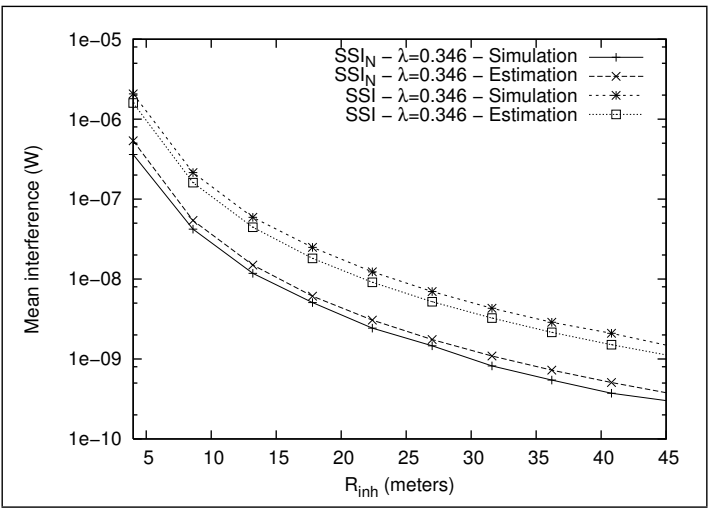

(a) Mean interference estimations.

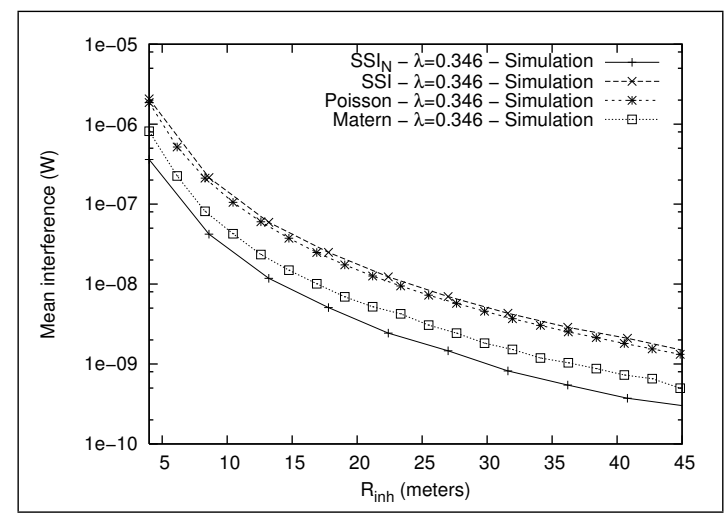

(b) Mean interference.

Figure 7: Mean interference estimations vs simulations.

\begin{tabular}{|c|c|c|c|}
\hline \multicolumn{4}{|c|}{ Effective transmitters intensity $(\mu)$} \\
\hline Poisson & Matérn & $S S I$ & $S S I_{N}$ \\
\hline \hline$\mu_{P}$ & $\mu_{M} \approx \frac{1}{\pi \cdot R_{i n h}^{2}}$ & $\mu_{S S I} \approx \frac{4 \cdot c}{\pi \cdot R_{i n h}^{2}}$ with $c \approx 0.56$ & $\mu_{S S I_{N}} \approx \frac{4 \cdot c_{N}}{\pi \cdot R_{i n h}^{2}}$ with $c_{N} \approx 0.18$ \\
\hline
\end{tabular}

Table 3: Mean intensity of effective transmitters according to the different point processes. 


\begin{tabular}{|c|c|c|c|}
\hline \multicolumn{5}{|c|}{ Mean interference intensity $\left(\mathbb{E}\left[I_{\Phi}(0)\right]\right)$} \\
\hline Poisson & Matérn & $S S I$ & $S S I_{N}$ \\
\hline \hline$\mu_{P} \cdot \int_{\Omega} l(\|x\|) d x$ & $\frac{1}{\pi \cdot R_{\text {inh }}^{2}} \cdot \int_{\Omega} l(\|x\|) d x$ & $\frac{4 \cdot c}{\pi \cdot R_{\text {inh }}^{2}} \cdot \int_{\Omega} l(\|x\|) d x$ & $\frac{4 \cdot c_{N}}{\pi \cdot R_{\text {inh }}^{2}} \cdot \int_{\Omega} l(\|x\|) d x$ \\
\hline
\end{tabular}

Table 4: Mean interference stemmed from effective transmitters according to the different point processes.

In the previous sections, different approaches have been proposed to thin a Poisson process according to the CSMA/CA modes. We have observed how the spatial distribution of simultaneous emitters may be influenced by these selection rules. We now investigate how these point processes may lead to different interference statistics.

\subsection{Simulation scenarios}

Albeit some analytical results are available for the Matérn process [33, 7], no analytical result is yet affordable for the S S I model. Therefore, most of the figures and conclusions presented in this section are drawn from simulation results. We give here the simulation setup.

\begin{tabular}{|l|l||l|l|}
\hline 802.15.4 868MHz Parameters & Numerical Values & Simulation parameters & Numerical values \\
\hline \hline Frequency & $868 \mathrm{MHz}$ & $\mathrm{R}$ & 100.0 meters \\
Wavelength & $\lambda=0.346$ meters & Radius of inhibition & 14.9 meters \\
Energy Detection Threshold $(\theta)$ & $-82 \mathrm{dBm}$ & $\mathrm{N}$ (except for Poisson) & 1500 \\
Emission power & $1 \mathrm{~mW}$ & Number of samples & at least 200, 000 \\
$\beta$ (path-loss parameter) & 3.0 & Path-loss function & $l(u)=\min \left(1,\left(\frac{\lambda}{4 \cdot \pi \cdot u}\right)^{\beta}\right)$ \\
\hline
\end{tabular}

Table 5: Simulation parameters.

To fit with realistic assumptions, we have considered the parameters of a $802.15 .4868 \mathrm{Mhz}$ interface [31] as described in Table 5. We consider the transmission of a frame between an emitter, denoted $Y$, and a receiver located at the origin $O$ and denoted $X_{0}$. The interference distribution is evaluated for this node, i.e. at the origin. In the rest of this paper, we assume that:

1. $R_{\text {inh }}$ is the inhibition radius defined as the distance for which the signal strength of a transmission is equal to the Energy Detection threshold (ED) denoted $\theta$. It is defined according to $\theta=l\left(R_{i n h}\right) \cdot P_{E}$.

2. the emitter $Y$ is located at a distance $\frac{R_{\text {inh }}}{2}$.

Two scenarios with the following features are considered:

1. The first scenario stands for the reference case. No RTS/CTS is used. $Y$ has sensed the medium clear and transmits data. Therefore potential transmitters in the vicinity of the transmitter are kept off. The interference 
at the origin is computed as the sum of interfering signals generated by all transmitters except $Y$. Let $\Phi$ be the point process modeling the set of interfering nodes, one gets:

$$
I_{\Phi}(0)=\sum_{X_{i} \in \Phi} P_{E} \cdot l\left(\left\|X_{i}\right\|\right)
$$

2. Scenario 2 models a transmission with RTS/CTS. The RTS/CTS mechanism is introduced in the point process model by considering that the receiver at the origin has also sensed a clear medium before the transmission and has transmitted a CTS. Therefore potential transmitters in the vicinity of the transmitter and the receiver are kept off. Thus, the emitter selection process differs from the first scenario but the final computation of interference still relies on 8 .

The different scenarios impact the Matérn and $S S I_{N}$ point processes regarding the set of nodes that are considered in the first step of the selection process. According to scenario $1, \Phi_{M}(0)=\{Y\}$ and according to scenario $2, \Phi_{M}(0)=$ $\left\{Y, X_{0}\right\}$

\subsection{First moment of interference distribution}

We now start by investigating the first moment of the interference distribution. We evaluate it as:

$$
\mathbb{E}\left[I_{\Phi}(0)\right]=\mu \cdot \int_{\Omega} l(\|x\|) d x
$$

$\Omega$ is the observation area where emitters are distributed: $\Omega=B(0, R) \backslash B\left(Y, R_{\text {inh }}\right)$ for scenario $1, \Omega=B(0, R) \backslash$ $\left(B\left(Y, R_{i n h}\right) \cup B\left(0, R_{i n h}\right)\right)$ for scenario 2 . In this formula, $\mu$ stands for the intensity of the considered point process.

It follows that the mean interference value relies on the average path-loss over $\Omega$ and on the point process intensity. Therefore, The problem boils down to the estimation of the intensity which was studied in the previous section for

the $S S I_{N}$ and known for the Poisson and Matèrn point process $\left(\mu_{M} \approx \frac{1}{\pi \cdot R_{i n h}^{2}}[33]\right)$. The intensities for the four point processes are summarized in Table 4

The mean interference for Scenario 1 is plotted in Figure 7(a) as a function of $R_{\text {inh }}$, or equivalently function of $\theta$. The difference between the simulation curves and the approximation proposed by formula (9) is tight. We can guess that the small difference is probably due to edge effects introduced by the presence of $Y$ and the boundary of $B(0, R)$.

In Figure 7(b), we plot the mean interference for the four point processes. There is no limit for the intensity of the 
Poisson process as for the $S S I$ or Matèrn point processes. In order to get results comparable to the $S S I$, we choose a Poisson intensity equal to the one of the $S S I$. For the two other point processes, Matèrn and $S S I_{N}$, we observe a significant difference.

The mean interference intensities corresponding to the different point processes are summarized in Table 4

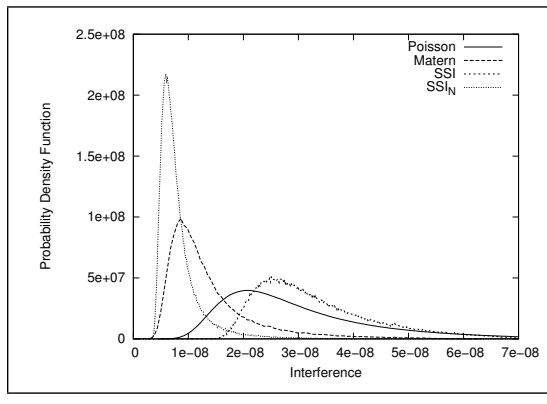

(a) Scenario 1 .

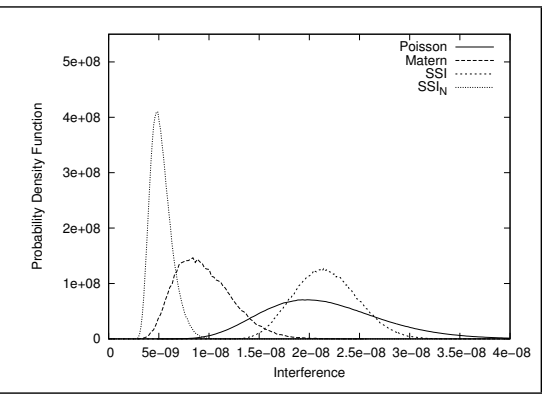

(b) Scenario 2 .

Figure 8: For a given scenario, comparison of interference $p d f$ between the point processes.

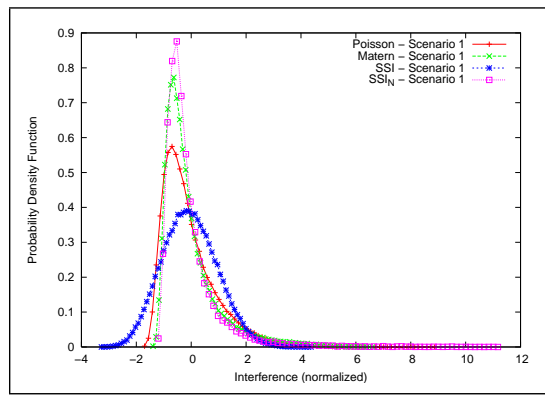

(a) Scenario 1 .

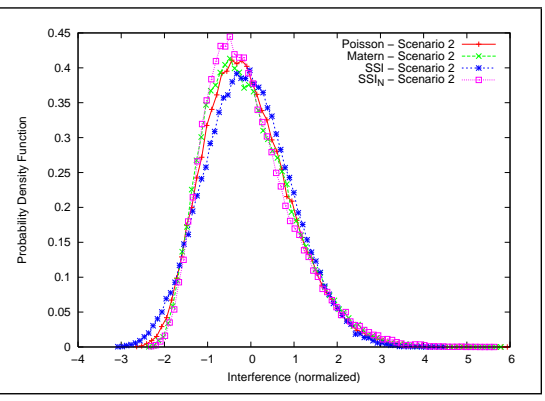

(b) Scenario 2 .

Figure 9: For a given scenario, comparison of interference $p d f$ between the point processes. The samples have been normalized.

\subsection{Interference $p d f$}

We now consider the probability density functions $(p d f)$ of interference at the origin as obtained with the different point processes and scenarios. These $p d f$ were assessed by simulations using the set of simulation parameters given in Table 5. For all the point processes, except for the Poisson one, the number of potential emitters $N$ is chosen such that no more active emitter can be added to the point process, i.e. after saturation. For the Poisson point process, the intensity is chosen equal to the $S S I$ intensity. Such configurations correspond to dense networks where a high number 


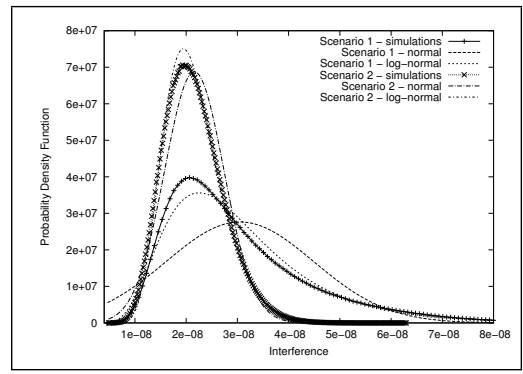

(a) Poisson - Scenarios 1 and 2 .

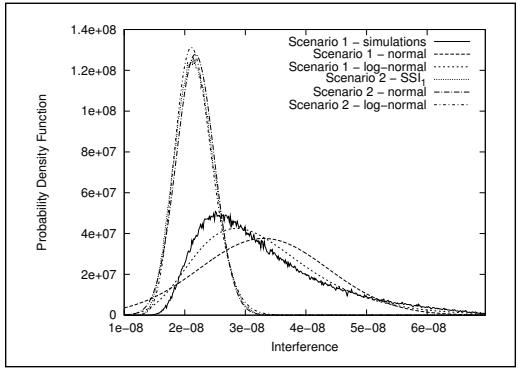

(b) $S S I$ - Scenarios 1 and 2 .

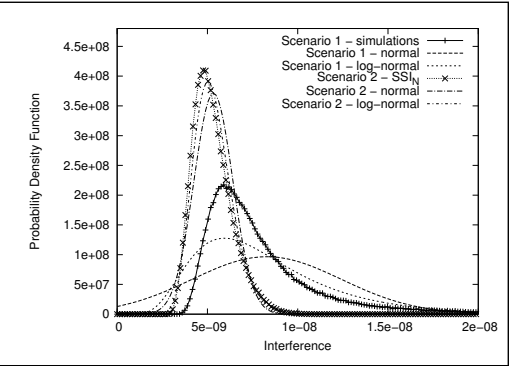

(c) $S S I_{N}-$ Scenarios 1 and 2.

Figure 10: Comparison of interference $p d f$ with normal and log-normal distributions.

of nodes compete for the medium.

Comparison between the point processes. In Figures $8(\mathrm{a})$ and $8(\mathrm{~b})$, we compare the interference $p d f s$ for the 4 point processes and the two scenarios. As we can see, the various point processes offer largely different interference $p d f s$ though they all offer a common shape: a peak and an asymmetry with a more or less heavy tail depending on the point process. The $p d f s$ seem to follow different distributions and offer different means and variances. In order to verify that they are not related to the same family of distributions, we compare these $p d f s$ again after normalizing the samples.

The normalized distributions are plotted in Figures 9(a) and 9(b) For scenario 1, the distributions are clearly different. For scenario 2, the $p d f s$ are very close to each others. Indeed, as we shall show, in this scenario, the different distributions get close to a log-normal distribution. In consequence, the normalization of the samples leads logically to distributions which offer a good similarity to a log-normal distribution with mean 0 and variance 1.

Comparison with normal and log-normal distributions. In Figures 10(a) to 10(c), we compare the interference pdf with normal and log-normal distributions. By lack of place, we plot only the interference $p d f$ for the Poisson, $S S I$ and $S S I_{N}$ point processes. It is clear, that for scenario 1, neither normal nor log-normal distributions fit, even if the log-normal offers a better approximation. For scenario 2, the distributions fit better. According to the statistics of a $\chi^{2}$ and a Smirnov-Kolmogorov test with 0.05 significance level, the log-normal is the best approximation for Poisson and $S S I_{N}$ point processes. The only exception is for the $S S I$ scenario 2 , where the interference $p d f$ is closer to a normal distribution. 
Discussion. From the results presented here, we can argue that the Poisson point process can hardly model CSMA/CA dense networks. Indeed, it leads to transmitter spatial locations and interference distributions which largely differ from the simulations and more realistic point processes such as the $S S I_{N}$ one.

However, much of the properties that can be computed with a Poisson point process are not tractable with the $S S I_{N}$ one. To bypass this difficulty, a solution is to use a log-normal law to model the interference distribution in CSMA/CA networks as proposed in Section7 The law parameters, its mean value and variance, can be chosen as the ones obtained with the $S S I_{N}$ modeling of transmitters. Based on the tractable log-normal law, it becomes possible to study more complex properties of the radio link such as the Bit Error Rate distribution of the Frame Error Rate, but also the capacity of the network considering the fact that links are unreliable and prone to interference.

\section{Conclusion}

In this article, we have argued that the $S S I_{N}$ point process offers an interesting opportunity to adequately model the location of transmitters in CSMA/CA wireless networks contrarily to traditional point processes such as the Poisson and Matérn ones. Indeed, the Poisson point process does not respect the spatial correlation induced by the CCA rules between simultaneous emitters. Moreover, the Poisson-based modeling does not provide any hint on the intensity of effective transmitters in the network. And even if the intensity is set artificially, it results in unrealistic variances. Hardcore point processes offer this information when reaching saturation. Under saturation, their intensity corresponds to the maximum number of simultaneous transmitters that can be reached in the network under the CCA rules. Among hard-core point processes, though the Matérn point process seems realistic, it suffers flaws such as a spatial anomaly which results in an under-estimation in the number of transmitters and in the resulting interference level.

Ultimately, the $S S I_{N}$ point process seems the most suited to offer a realistic modeling of the different CCA modes of CSMA/CA based multi hop wireless networks. This assumption is empirically proven by the study of the intensity and the network capacity. We have shown, through simulations and for different kind of ad hoc networks (classical ad hoc, mesh and sensor networks) that the number of simultaneous transmitters fits to the $S S I_{N}$ intensity. These intensities may be easily deduced from the CSMA/CA parameters. Therefore, in CSMA/CA networks, it seems that the number of frames that can be transmitted per second is proportional to the intensity of the adequate $S S I_{N}$. This total capacity must be divided by the mean number of hops between sources and destinations and the number of 
source-destination pairs. A comparison between this theoretical capacity estimation and the one obtained through extensive simulations show similar result. It validates the relevance of our approach and empirically confirms that the capacity is $O\left(\frac{1}{n}\right)$ in CSMA/CA multi hop wireless networks.

The future works now consist in studying further properties of the radio link using a log-normal law to model the interference distribution in the CSMA/CA network. It should provide us with the perspective to study the capacity of dense CSMA/CA networks under the hypothesis that links are subject to interference and thus are unreliable.

\section{References}

[1] Network simulator 3 - ns3. http: / / www. nsnam.org.

[2] F. Baccelli, B. Błaszczyszyn, and P. Mühlethaler. An aloha protocol for multihop mobile wireless networks. IEEE Transactions on Information Theory, 52(2):421-436, 2006.

[3] F. Baccelli, B. Blaszczyszyn, and P. Muhlethaler. On the performance of time-space opportunistic routing in multihop mobile ad hoc networks. In 6th International Symposium on Modeling and Optimization in Mobile, Ad Hoc, and Wireless Networks and Workshops, 2008. WiOPT 2008., Berlin, Germany, April 2008. IEEE.

[4] E. Ben Hamida, G. Chelius, A. Busson, and E. Fleury. Neighbor discover in multi-hop wireless networks: evaluation and dimensioning with interference considerations. Discrete Mathematics and Theoretical Computer Science, 10(2):87-14, 2008.

[5] B. Blaszczyszyn and D. Yogeshwaran. Connectivity in sub-poisson networks. In 48th Annual Allerton Conference on Communication, Control, and Computing, Allerton, USA, April 2010. SIAM.

[6] A. Busson and G. Chelius. Point processes for interference modeling in csma/ca ad-hoc networks. In Sixth ACM International Symposium on Performance Evaluation of Wireless Ad Hoc, Sensor, and Ubiquitous Networks (PE-WASUN 2009), Tenerife, Spain, October 2009. IEEE.

[7] A. Busson, G. Chelius, and J-M. Gorce. Interference modeling in csma multi-hop wireless networks. Research Report RR-6624, INRIA, September 2008. 
[8] T.G. Cheng, Y.-C.; Robertazzi. A new spatial point process for multihop radio network modeling. In SUPERCOMM/ICC, 1990.

[9] B. Cho. A Simulation study on Interference in CSMA/CA Ad-Hoc Networks using Point Process. Master thesis, Aalto University - School of Science and Technology, Dec 2010.

[10] M. Conti and S. Giordano. Multihop ad hoc networking: The reality. IEEE Communications Magazine, 45(4):8895, 2007.

[11] D.J. Daley and D. Vere-Jones. An Introduction to the theory of point processes. Springer-Verlag, New York, USA, 2003.

[12] O. Dousse, F. Baccelli, and P. Thiran. Impact of interferences on connectivity in ad hoc networks. IEEE/ACM Transactions on Networking, 13(2):425-436, April 2005.

[13] O. Dousse and P. Thiran. Connectivity vs capacity in dense ad hoc networks. In Conference on Computer Communications (INFOCOM), Hong Kong, China, March 2004. IEEE.

[14] O. Dousse, P. Thiran, and M. Hasler. Connectivity in ad-hoc and hybrid networks. In Conference on Computer Communications (INFOCOM), New York, USA, June 2002. IEEE.

[15] M. Franceschetti, O. Dousse, D. Tse, and P. Thiran. Closing the gap in the capacity of wireless networks via percolation theory. IEEE Transactions on Information Theory, 53(3):1009-1018, 2007.

[16] Radha Krishna Ganti and Martin Haenggi. Spatial and temporal correlation of the interference in aloha ad hoc networks. IEEE Communications Letters, 13(9):631-633, 2009.

[17] P. Gupta and P. Kumar. Capacity of wireless networks. IEEE Transactions on Information Theory, 46(2):388404, 2000.

[18] P. Hall. Introduction To the Theory of Coverage Processes. Wiley, 1988. 
[19] J. Hoydis, M. Petrova, and P. Mahonen. Effects of topology on local throughput-capacity of ad hoc networks. In IEEE 19th International Symposium on Personal, Indoor and Mobile Radio Communications, 2008. PIMRC 2008., Cannes, France, September 2008. IEEE.

[20] Huang Kaibin, V.K.N. Lau, and Yan Chen. Spectrum sharing between cellular and mobile ad hoc networks: transmission-capacity trade-off. IEEE Journal on Selected Areas in Communications, 27(7):631-633, 2009.

[21] B. Lauwens, B. Scheers and A. Van de Capelle. Performance analysis of unslotted csma/ca in wireless networks. Telecommunication Systems, 44(1-2):109-123, 2010.

[22] H. W. Lotwick. Simulations of some spatial hard-core models, and the complete packing problems. Journal of Statistical Computation and Simulation, 15:295-314, 1982.

[23] B. Matérn. Spatial variation. Meddelanden fran Statens Skogsforskningsinstitut, 49(5):1-144, 1960.

[24] B. Matérn. Spatial variation. Lecture Notes in Statistic 36. Springer Verlag, Berlin, Heidelberg, New York, 1986.

[25] V. Mhatre, C. Rosenberg, and R. Mazumdar. The capacity of random ad hoc networks under a realistic link layer model. IEEE Transactions on Information Theory, 55(6):2494-2498, 2009.

[26] H.Q. Nguyen, F. Baccelli, and D. Kofman. A stochastic geometry analysis of dense ieee 802.11 networks. In Conference on Computer Communications (INFOCOM), Anchorage, USA, May 2007. IEEE.

[27] I. Palasti. On some random space filling problem. Publications of Mathematical Institute of Hungarian Academy of Sciences, 5(1):353-359, 1960.

[28] D. H. Park, C. G. Han and S. J. Ahn. Performance analysis of mac layer protocols in the ieee 802.11 wireless lan. Telecommunication Systems, 33(1-3):233-253, 2006.

[29] S. Saunders. Antennas and propagation for wireless communication systems. Wiley, 1999.

[30] IEEE Computer Society. Ieee standard for information technology - telecommunications and information exchange between systems - local and metropolitan area networks - specific requirements - part 11: Wireless lan 
medium access control (mac) and physical layer (phy) specifications: High-speed physical layer in the 5ghz band. Technical report, IEEE Computer Society, 1999.

[31] IEEE Computer Society. Ieee standard for information technology - telecommunications and information exchange between systems - local and metropolitan area networks - specific requirements - part 15.4: Wireless medium access control (mac) and physical layer (phy) specifications for low-rate wireless personal area networks (wpans). Technical report, IEEE Computer Society, 2006.

[32] H. Solomon and H. Weiner. A review of the packing problem. Communications in Statistics and Theoretical Methods, 15:2571-2607, 1986.

[33] D. Stoyan, W. Kendall, and J. Mecke. Stochastic Geometry and Its Applications, 2nd Edition. John Wiley and Sons Ltd, Chichester, UK, 1996.

[34] K. Szczypiorski and J. Lubacz. Saturation throughput analysis of ieee 802.11g (erp-ofdm) networks. Telecommunication Systems, 38(1-2):45-52, 2008.

[35] M. Tahir and S. K. Mazumder. Markov chain model for performance analysis of transmitter power control in contention based wireless mac protocol. Telecommunication Systems, 38(3-4):99-110, 2008.

[36] M. Tanemura. On random complete packing by disks. Annals of the Institute of Statistical Mathematics (AISM), $31: 351-365,1979$.

[37] X. Yang and A. P. Petropulu. Co-channel interference modeling and analysis in a poisson field of interferers in wireless communications. IEEE Transactions on Signal Processing, 51(1):64-76, January 2003. 